\title{
Seqüestro pulmonar extralobar: análise anatomopatológica de dois casos em natimortos e revisão da literatura
}

\section{Extralobar pulmonary sequestration: anatomical analysis of two cases in stillbirths and review of literature}

Ítalo Martins de Oliveira'; Davi Tunde Câmara Opaleye²; José Felipe Santiago Jr.3; Augusto Tadeu Barros de Sousa ${ }^{3}$; Rafael Siqueira Athayde Lima ${ }^{4}$; Maria do Patrocínio Ferreira Grangeiro ${ }^{5}$; Dalgimar Beserra de Menezes ${ }^{6}$; Carlos Augusto Alencar Jr. ${ }^{7}$

\section{unitermos \\ Seqüestro pulmonar \\ Malformações congênitas \\ Necropsia}

\section{resumo}

O seqüestro pulmonar é definido como uma massa anormal de tecido pulmonar sem comunicação com a árvore brônquica. É anomalia rara, responsável por 0,15-6,45\% das malformações pulmonares congênitas. Quando possui revestimento pleural próprio, chama-se seqüestro pulmonar extralobar (SPE). Este trabalho descreve dois casos de SPE em natimortos (NM) com 32 (1) e 34 (2) semanas de gestação com diagnóstico clínico de hipoxia intra-uterina e adenomatose cística, respectivamente, e faz revisão da literatura. O diagnóstico envolveu análise ultra-sonográfica, sindrômica, macroscópica e microscópica dos NM. Foi observada massa supradiafragmática no hemitórax esquerdo ligada à aorta torácica (1) e ao diafragma (2). As MFs associadas foram agenesia tímica (2), hipoplasia pulmonar (2), pé torto congênito (1) e acondroplasia de membros (2). A microscopia evidenciou, nos dois casos, tecido pulmonar imaturo e pedículo vascularizado e inervado.

\section{abstract}

Pulmonary sequestration represents an abnormal pulmonary mass that does not communicate with the tracheobronchial tree. It is a rare malformation (MF), accountable for $0.15 \%-6.45 \%$ of pulmonary congenital MFs. When it has its own pleural covering, it is called extralobar (EBPS). This work describes two cases of EBPS in stillbirths (SB), at 32 (1) and 34 (2) weeks' gestation, with clinical diagnosis of intrauterine hypoxia and cystic adenomatosis, respectively. It also reviews the literature on the subject. The diagnosis involved ultrasonographic, syndromic, macroscopic and microscopic analysis. The macroscopy showed a supradiaphragmatic mass in the left hemithorax linked to thoracic aorta (1) and diaphragm (2). The associated MFs were: thymic agenesis (2), pulmonary hypoplasia (2), clubfoot (1) and achondroplasia (2). Microscopy demonstrated, in both cases, immature pulmonary tissue and vascularized and innervated pedicle.

\section{key words}

Pulmonary sequestration

Congenital malformation

Necropsy

\footnotetext{
1. Bolsista do Programa Institucional de Bolsas de Iniciação Cientifica do Conselho Nacional de Desenvolvimento Cientifico e Tecnológico (PIBIC-CNPq) no período da pesquisa; pós-graduando do Programa de Doutorado em Cardiologia do Departamento de Cardiopneumologia da Faculdade de Medicina da Universidade de São Paulo (FMUSP).

2. Médico residente de Geriatria do Hospital das Clínicas da USP.

3 Médicos graduados na Faculdade de Medicina da Universidade Federal do Ceará (FM/UFC).

4. Aluno da graduação da FM/UFC.

5. Patologista do Departamento de Patologia e Medicina Legal da UFC.

6. Patologista do Departamento de Patologia e Medicina Legal da UFC; livre-docente pela Universidade Estadual do Ceará (UECE).

7. Professor adjunto do Departamento de Medicina Materno-Infantil da UFC.
} 


\section{Introdução}

Seqüestro pulmonar é uma anomalia congênita incomum, definida como uma massa de tecido pulmonar que apresenta conexão anormal com a árvore traqueobrônquica ${ }^{(4,6,9)}$. Denomina-se extralobar quando possui revestimento pleural próprio, e intralobar quando se apresenta revestido pela pleura visceral de um lobo pulmonar normal|(10). O seqüestro pulmonar apresenta irrigação por uma artéria sistêmica anômala e tem drenagem para veias sistêmicas (geralmente na forma extralobar) ou pulmonares (geralmente na forma intralobar) ${ }^{(4,5,9,10)}$.

O seqüestro pulmonar é mais freqüente no sexo masculino (4:1) e, na grande maioria das vezes, é unilateral, predominando à esquerda $(80 \% \text { dos casos) })^{(5,10)}$. O seqüestro pulmonar extralobar (SPE) é comumente associado a outras anomalias congênitas, sendo os defeitos diafragmáticos encontrados em cerca de $50 \%$ dos $\operatorname{casos}^{(5)}$. Este trabalho relata dois casos de SPE em natimortos (NM) e faz uma revisão da literatura.

\section{Relatos de caso}

\section{Caso 1}

Paciente de 25 anos, G1P0A0, encaminhada ao serviço de obstetrícia da Maternidade-Escola Assis Chateaubriand da Universidade Federal do Ceará (UFC) com hipótese diagnóstica de poliidrâmnio e doença hipertensiva específica da gestação (DHEG), apresentando picos hipertensivos e edema de extremidades após o início da gravidez. Relatou cefaléia frontal e tontura; não havia qualquer antecedente obstétrico mórbido e referiu como antecedentes familiares: hipertensão arterial sistêmica (HAS), cardiopatia e gemelaridade. Negou ocorrência de malformações na família, alergias e casamento consangüíneo, bem como antecedentes de afecções pessoais relevantes, exposição a agentes teratogênicos, etilismo, tabagismo e uso de drogas ilícitas ou medicamentos.

Foi realizado exame ultra-sonográfico obstétrico para esclarecimento diagnóstico, sendo visualizado feto único do sexo feminino, na 32a semana de gestação, com apresentação cefálica e posição esquerda, movimentos cardíacos rítmicos, poliidrâmnio (índice de líquido amniótico [ILA] = 238) e ausência de movimentos respiratórios. Na dopplervelocimetria evidenciaram-se diástole zero de artéria umbilical e incisura protodiastólica rasa da artéria uterina esquerda. A cardiotocografia constatou bradicardia fetal, diagnosticando-se sofrimento fetal agudo, sendo indicado parto abdominal de urgência, sem sucesso. O NM de 1.810 $\mathrm{g}$ foi encaminhado para estudo anatomopatológico após assinatura do termo de consentimento livre e esclarecido pela mãe.

O NM apresentou protrusão do globo ocular e pé torto congênito esquerdo. O exame interno demonstrou pulmão esquerdo hipoplásico e massa no terço inferior do hemitórax ipsilateral ligada indiretamente à aorta abdominal por pedículo diafragmático (Figura 1). O estudo microscópico desta massa evidenciou tecido pulmonar imaturo (Figura 2). $\mathrm{O}$ pedículo apresentava veias, uma artéria de médio calibre e terminações nervosas (Figura 3).

\section{Caso 2}

Paciente de 21 anos, G2P1A0, procurou o Serviço de Obstetrícia do Hospital Geral de Fortaleza para elucidação de malformação ao exame ultra-sonográfico de rotina

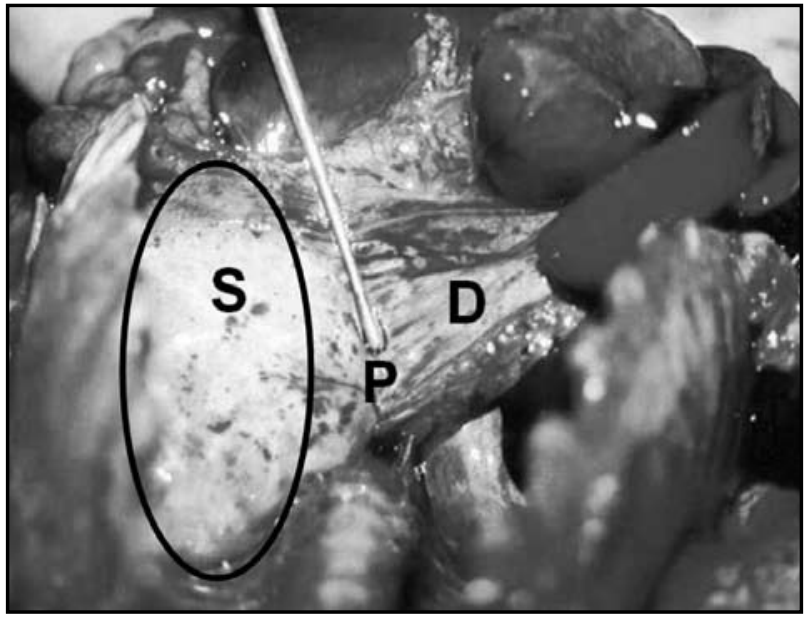

Figura 1 - Aspecto macroscópico do caso 1 em visão cefalocaudal S: seqüestro pulmonar extralobar (elipse); P: pedículo ligado ao diafragma; D: diafragma.

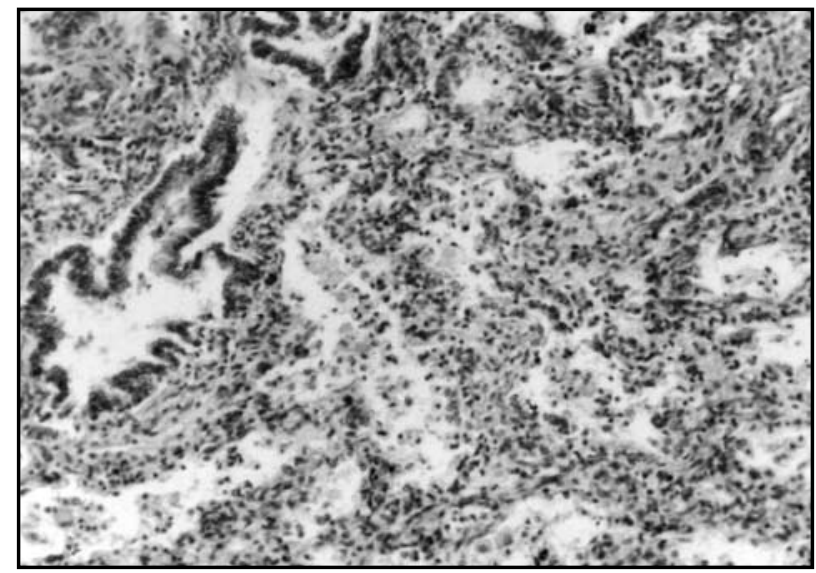

Figura 2 - Microscopia (HE 100x) do seqüestro pulmonar do caso 1 


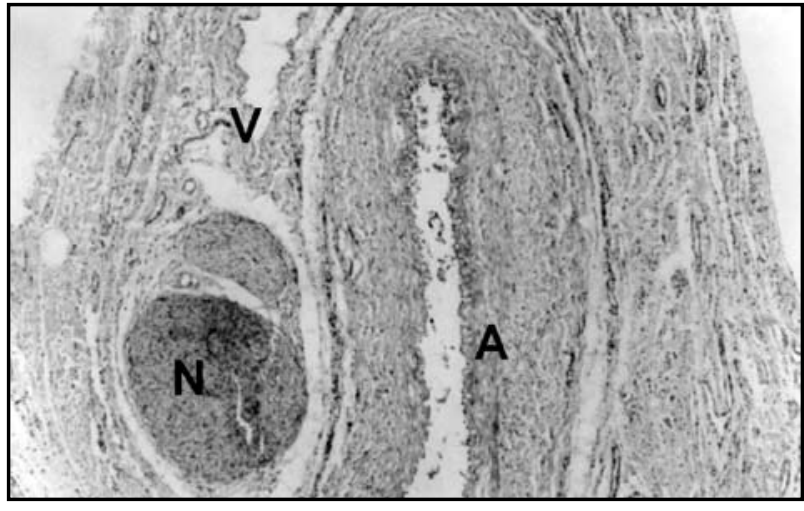

Figura 3 - Microscopia (HE 60x) do pedículo do seqüestro pulmonar do caso 1 N: filetes nervosos; A: artéria de médio calibre; V: veia.

realizado na 31a semana de gravidez. A paciente referiu, como antecedente obstétrico, óbito de recém-nascido pré-termo de 30 semanas de gestação. Negou antecedentes mórbidos pessoais ou familiares, exposição a agentes teratogênicos, etilismo, tabagismo e utilização de drogas ilícitas ou medicamentos.

Realizou-se nova ultra-sonografia (US) para esclarecimento diagnóstico, na qual visualizou-se feto único do sexo masculino com 34 semanas de gestação, apresentação pélvica, evidenciando massa não-pulsátil no terço inferior do hemitórax esquerdo compatível com malformação adenomatosa cística III. O exame constatou, ainda, hidropisia fetal, desvio do mediastino para a direita, derrame pleural bilateral, ascite volumosa e varicocele.

Durante o acompanhamento hospitalar realizaram-se toracocentese fetal de alívio (85 $\mathrm{ml}$ de líquido citrino) e ciclo de corticoterapia para maturação pulmonar. No quarto dia de internamento foi detectado sofrimento fetal à cardiotocografia, indicando-se parto abdominal de urgência, sem sucesso. O NM de $3.400 \mathrm{~g}$ foi encaminhado para estudo anatomopatológico após a assinatura do termo de consentimento livre e esclarecido pela mãe.

O NM apresentou fácies sindrômica, assimetria torácica com abaulamento do hemitórax direito, anasarca e acondroplasia dos membros superiores e inferiores. O exame interno evidenciou congestão e edema cerebral, ascite de $50 \mathrm{ml}$, agenesia tímica, cavidade torácica com derrame pleural bilateral de $50 \mathrm{ml}$, hipoplasia pulmonar bilateral e massa no terço inferior do hemitórax esquerdo ligada à aorta torácica por pedículo preso ao diafragma (Figura 4). O estudo microscópico evidenciou órgãos fetais compatíveis com a idade gestacional e SPE, com presença de tecido pulmonar ora maduro, ora imaturo.

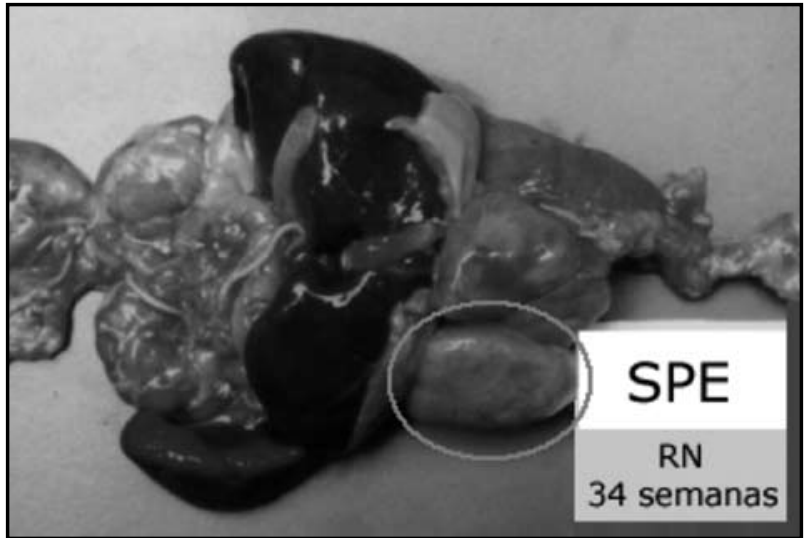

Figura 4 - Aspecto macroscópico do caso 2

Seta: seqüestro pulmonar extralobar.

\section{Discussão}

O seqüestro pulmonar é caracterizado por uma massa pulmonar não-funcionante, sem comunicação normal com a árvore brônquica e vascularizada por uma artéria sistêmica ${ }^{(4,6)}$. Representa anomalia rara, responsável por 0,15\% a 6,45\% das malformações pulmonares congênitas ${ }^{(5)}$. A irrigação arterial anômala para um tecido pulmonar foi descrita inicialmente por Huber (1777) $)^{(4)}$. Rokitansky e Rektorzic (1861) propuseram ser o seqüestro oriundo de uma fração pulmonar separada durante a organogênese ${ }^{(4,5)}$. Price (1946) foi o primeiro a empregar o termo "seqüestro" e identificar o peculiar achado de um suprimento arterial aberrante. Esta teoria é comumente aceita como a melhor explicação etiopatogênica dessa malformação( ${ }^{(4,5,8)}$.

A classificação em SPE ou seqüestro pulmonar intralobar baseia-se na existência de um envoltório pleural próprio. A forma extralobar, freqüentemente localizada nos lobos pulmonares inferiores, nos espaços costodiafragmático e infradiafragmático, possui um envoltório pleural próprio(3-5,10).

O seqüestro pulmonar intralobar é envolto por parênquima pulmonar normal maduro, não possui envoltório pleural e geralmente é encontrado no segmento póstero-lateral do pulmão esquerdo ${ }^{(2,5,8)}$. Nesse caso, é ocasionalmente encontrada intercomunicação com a árvore pulmonar adjacente, provavelmente via poros de $\mathrm{Kohn}^{(5,8)}$.

A forma extralobar compreende $25 \%$ de todos os casos de seqüestro pulmonar, tem maior prevalência no sexo masculino (4:1) e em $80 \%$ dos casos se localiza à esquerda do tórax. Já o seqüestro pulmonar intralobar é igualmente distribuído tanto em relação ao sexo, quanto ao lado acometido ${ }^{(2,5)}$. Savic et al. ${ }^{(9)}$ observaram 133 casos de SPE contra 400 casos intralobares em uma extensa revisão da literatura. 
Em aproximadamente $50 \%$ dos casos, o SPE coexiste com anomalias diafragmáticas, cardíacas ou digestivas, sendo a hérnia diafragmática a mais comum (30\% a 65\% dos (asos) $)^{(5)}$. As demais anomalias associadas são: atresia bronquial, duplicação colônica ou ileal, conexão esofágica, anomalias das vértebras cervicais e defeitos pulmonares, incluindo hipoplasia. Na forma intralobar, a associação com outras malformações é menor, cerca de $14 \%^{(2-4)}$.

A irrigação do seqüestro pulmonar é suprida, em $80 \%$ dos casos, pela aorta descendente. As artérias gástrica, esplênica e subclávia são citadas como outras fontes de suprimento arterial. O calibre do vaso nutriz é geralmente maior no seqüestro pulmonar intralobar, contrastando com 0 aspecto delgado no $S P E^{(5)}$. A drenagem é feita pelas veias pulmonares na forma intralobar e por tributária das veias cava superior, ázigos e porta na forma extralobar ${ }^{(5)}$.

O SPE geralmente é diagnosticado na infância, enquanto o intralobar pode ter curso indolente até a segunda década de vida, usualmente se manifestando sob a forma de pneumonias recorrentes ${ }^{(2,6)}$. Diagnóstico tardio e evidências histopatológicas reforçam a teoria proposta de que essa forma de seqüestro pulmonar é de origem adquirida, oriunda de um processo inflamatório crônico de natureza infecciosa ${ }^{(4)}$. Não há relação descrita com qualquer alteração cromossomial.

A US pré-natal geralmente visualiza uma massa homogênea ecodensa de limites definidos ${ }^{(1,5)}$. $\mathrm{O}$ achado ao doppler de uma aferência arterial oriunda da aorta torácica ou do tronco celíaco é patognomônico de seqüestro ${ }^{(3,5)}$. Uma malformação cística adenomatosa pode aparecer de forma semelhante sem apresentar o suprimento arterial, o que sugere origem embriológica comum ${ }^{(5)}$. A investigação ultra-sonográfica nessa época da vida é também importante para a identificação de poliidrâmnio e hidropisia fetal, importantes preditores de prognóstico fetal ${ }^{(2)}$.
Uma imagem radiológica do tórax de aspecto cístico adjacente e, freqüentemente, posterior ao pericárdio é sugestiva de seqüestro pulmonar, porém não descarta outras lesões do mesmo aspecto, muito menos distingue entre as formas extralobar e intralobar. A existência de intercomunicações com o trato digestivo superior e, portanto, a identificação de malformações associadas a essa região podem ser demonstradas com exames contrastados ${ }^{(5)}$.

O método padrão-ouro para diagnóstico do seqüestro pulmonar é a aortografia. Entretanto, devido à boa acurácia e à menor agressividade da angiotomografia multislice e da angiorressonância, estes métodos também representam boas opções para o diagnóstico(2).

O tratamento cirúrgico depende intimamente da forma de apresentação dos casos e de malformações associadas. Nesse aspecto, a videotoracoscopia vem apresentando papel cada vez mais importante, com alguns resultados sugerindo menor agressão e recuperação mais rápida do paciente ${ }^{(4)}$.

Nos casos relatados, as anomalias associadas foram agenesia tímica, hipoplasia pulmonar e anomalias esqueléticas (pé torto congênito e acondroplasia). Não foram encontradas alterações diafragmáticas ou do trato gastrointestinal. Dada a raridade do seqüestro pulmonar, em especial o extralobar, o presente relato de caso registra casos atípicos em sua apresentação morfológica. Demonstra-se, assim, a importância dessa malformação no diagnóstico diferencial das patologias torácicas neonatais.

\section{Agradecimentos}

Agradecemos à Profa. Dra. Sílvia Helena Rabenhost e ao Prof. Dr. Paulo Roberto Carvalho de Almeida, pelo apoio científico, e ao funcionário José Ivan Rodrigues de Sousa, pela colaboração técnica.

\section{Referências}

1. ADZICK, N.S. et al. Fetal lung lesions: management and outcome. Am J Obstet Gynecol, v. 179, n. 4, p. 884-9, 1998.

2. BRATU, I. et al. The multiple facets of pulmonary sequestration. J Pediatr Surg, v. 36, n. 5, p. 784-90, 2001.

3. CARPENTIERI, D. F. et al. Subdiaphragmatic pulmonary sequestration: a case report with review of the literature. J Perinatol, v. 20, n. 1, p. 60-2, 2000.
4. GEZER, S. et al. Pulmonary sequestration: a singleinstitutional series composed of 27 cases. J Thorac Cardiovasc Surg, v. 133, n. 4, p. 955-9, 2007.

5. HALKIC, N. et al. Pulmonary sequestration: a review of 26 cases. Eur J Cardiothorac Surg, v. 14, n. 2, p. 127-33, 1998.

6. LOUIE, H. W. et al. Pulmonary sequestration: 17-year experience at UCLA. Am Surg, v. 59, n. 12, p. 801-5, 1993. 
7. NICOLETTE, L. A. et al. Intralobar pulmonary sequestration: a clinical and pathological spectrum. J Pediatr Surg, v. 28, n. 6, p. 802-5, 1993.

8. PICCIONI, W. J. et al. Pulmonary sequestration in the neonate. Chest, v. 97, n. 1, p. 244-6, 1990.

9. SAVIC, B. et al. Lung sequestration: report of seven cases and review of 540 published cases. Thorax, v. 34, n. 1, p. 96-101, 1979.

10. STERN, R. et al. Bilateral intralobar pulmonary sequestration in a newborn, case report and review of the literature on bilateral pulmonary sequestrations. J Pediatric Surg, v. 42, n. 4, p. 19-23, 2007 